\title{
The Reduction in Urinary Glutamate Excretion Is Responsible for Lowering Urinary pH in Pink Urine Syndrome
}

\author{
Susumu Ogawa, ${ }^{1,2}$ Junko Takiguchi, ${ }^{2}$ Manami Shimizu, ${ }^{1}$ Kazuhiro Nako, ${ }^{1}$ \\ Masashi Okamura, ${ }^{1}$ Yoshitaka Kinouchi ${ }^{2}$ and Sadayoshi Ito ${ }^{1}$ \\ ${ }^{1}$ Division of Nephrology, Endocrinology and Vascular Medicine, Tohoku University Hospital, Sendai, Miyagi, \\ Japan \\ ${ }^{2}$ Institute for Excellence in Higher Education, Division of Research in Student Support, Section of Clinical \\ Medicine, Tohoku University, Sendai, Miyagi, Japan
}

\begin{abstract}
We frequently encounter brownish-red, cloudy urine in some obese subjects, which occurs due to pink urine syndrome (PUS). PUS is a phenomenon in which uric acid precipitates into the urine due to reduced urinary $\mathrm{pH}(\mathrm{UpH})$. The mechanism underlying urinary acidification has not been elucidated so far. $\mathrm{UpH}$ level is adjusted by urinary excretion of ammonia synthesized from glutamate or glutamine, suggesting that renal synthesis of ammonia from glutamate or glutamine is decreased in PUS. However, this hypothesis has not been examined yet. We therefore examined the changes in the urinary excretion of these amino acids in PUS. One-hundred-fifty male students who had undergone a physical examination were enrolled. To determine the presence [PUS (+), $n=72$ ] or absence [PUS $(-), n=78$ ] of PUS, urinary amino acid excretion and $\mathrm{UpH}$ were evaluated. Independent risk factors of lower $\mathrm{UpH}$ were determined using multiple regression analyses. The PUS (+) subjects, who had lower UpH values than PUS (-) subjects, showed lower urinary excretion of glutamate and some other glucogenic amino acids. Thus, UpH correlated positively with the urinary excretion of glutamate in the PUS (+) subjects. A reduction in urinary glutamate but not in glutamine excretion proved to be an independent risk factor for reduced UpH. In conclusion, PUS appears to occur when a reduction in the synthesis of ammonia from glutamate causes a decrease in $\mathrm{UpH}$. Our results showed that urinary glutamate excretion was reduced in PUS because renal glutamate was consumed by a reaction different from ammonia production.
\end{abstract}

Keywords: ammonia; glucogenic amino acids; glutamate; pink urine syndrome; urinary $\mathrm{pH}$.

Tohoku J. Exp. Med., 2016 June, 239 (2), 103-110. (C) 2016 Tohoku University Medical Press

\section{Introduction}

While handling urine samples during health checkups, we frequently encounter brownish-red, cloudy urine, which, upon centrifugation, produces pink sediments that are either uric acid crystals or urates. This phenomenon is defined as pink urine syndrome (PUS) (Deitel et al. 1984; Ogawa et al. 2015b). PUS is characterized by cloudy brownish-red urine and the formation of pink sediments in urine upon centrifugation, which occurs due to uric acid (UA) precipitation resulting from urinary acidification. PUS is not a rare phenomenon, with a considerable number of PUS cases $(4.4 \%)$ found even in healthy young populations (Ogawa et al. 2015b); it is also closely linked to obesity (Deitel et al. 1984; Ogawa et al. 2015b). However, PUS is not a disease; instead, it appears to be a symptom that elicits some sort of physical response. However, the mechanism underlying the phenomenon of PUS is and its significant remains unclear. Not all obese patients manifest symptoms of PUS. In addi- tion, whether PUS develops in obese patients who fulfill certain criteria or whether the pathology that induces PUS may lead to obesity but does not necessarily cause it is not certain.

PUS results from increased urinary UA (UUA) excretion and a reduction in urinary $\mathrm{pH}(\mathrm{UpH})$ level. A reduction in UpH in PUS has been shown to be strongly correlated with oxidative stress (OS) markers in the urine (Ogawa et al. 2015b). UpH is controlled by the secretion of urinary ammonia $\left(\mathrm{NH}_{3}\right)$ from the renal proximal tubules (RPTs), where it is converted into ammonium ions $\left(\mathrm{NH}_{3}+\mathrm{H}^{+} \rightarrow\right.$ $\mathrm{NH}_{4}^{+}$) (Hems 1975; Zipp and Tannen 1983; RodriguezNichols et al. 1984). The UpH decreases when the supply of $\mathrm{H}^{+}$is so high (acidosis) that it cannot be counteracted by this $\mathrm{NH}_{3}$, or when the secretion of $\mathrm{NH}_{3}$ from the RPT decreases. Synthesis of $\mathrm{NH}_{3}$ in the RPTs involves two reactions: the production of glutamate from glutamine, and the production of $\alpha$-ketoglutarate from glutamate (Hems 1975; Zipp and Tannen 1983; Rodriguez-Nichols et al. 1984;

Received March 11, 2016; revised and accepted April 28, 2016. Published online May 26, 2016; doi: 10.1620/tjem.239.103.

Correspondence: Susumu Ogawa, M.D., Ph.D., Division of Nephrology, Endocrinology and Vascular Medicine, Tohoku University

Hospital, 1-1 Seiryo-machi, Aoba-ku, Sendai, Miyagi 980-8574, Japan.

e-mail: ogawa-s@hosp.tohoku.ac.jp 
Nissim 1999; Tapiero et al. 2002; Karim et al. 2005) (Fig. 1). After filtration in the renal glomeruli, amino acids (AAs) in the blood are reabsorbed and used in the RPT, with the remainder being excreted into the urine (Moret 2007). Therefore, the amount of AAs excreted into the urine might be an important marker that indicates the use of those AAs in the RPTs. Since the UpH is reduced in PUS, it is assumed that, because of some sort of impediment of the RPTs, the use of either glutamine or glutamate and $\mathrm{NH}_{3}$ synthesis is decreased, while the amount of urinary glutamine or glutamate excretion is likely to have increased. However, there are no reports so far to confirm this hypothesis.
We therefore measured the amount of AAs including glutamine and glutamate and the amount of UA excreted in the PUS $(+)$ subjects and investigated the relationship between $\mathrm{UpH}$ and the amount of urinary excretion of these AAs and UA.

\section{Subjects and Methods}

Subjects

The subjects enrolled for our previous study (Ogawa et al. 2015b) were used for this study as well. A large proportion of the study population were men, and since numerous cases of UA precipitation have been observed among men (Ogawa et al. 2015b), we decided to conduct our analysis using male subjects only. Of the

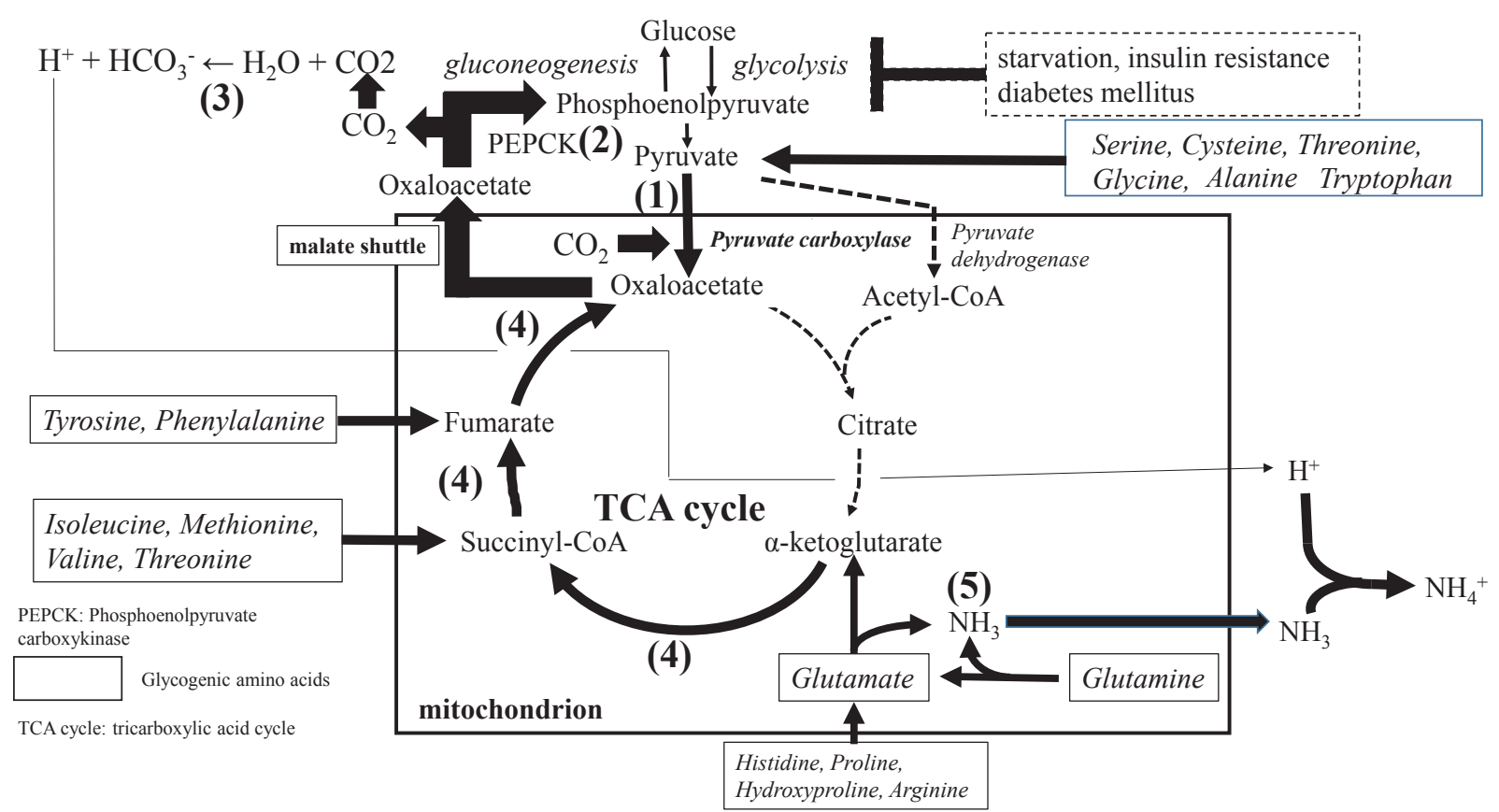

Fig. 1. The relationship, under acidotic conditions, between glucose metabolism and amino acids use in the renal proximal tubules.

The Figure 1 shows the relationship, under acidotic conditions, between glucose metabolism and glucogenic amino acids (GAAs) use in the renal proximal tubules (RPT).

Under conditions such as starvation, dehydration, insulin resistance and diabetes, the glycolysis decreases, so acidosis is liable to be induced. The roles that the kidneys play under acidotic conditions are to increase the urinary excretion of hydrogen $\left(\mathrm{H}^{+}\right)$, increase the production of bicarbonate $\left(\mathrm{HCO}_{3}{ }^{-}\right)$. The $\mathrm{HCO}_{3}^{-}$is produced by the reaction (3) $\left[\right.$water $\left(\mathrm{H}_{2} \mathrm{O}\right)$ + carbon dioxide $\left(\mathrm{CO}_{2}\right) \rightarrow \mathrm{H}^{+}+\mathrm{HCO}_{3}^{-}$. To move this reaction forward, it is necessary to eliminate $\mathrm{H}^{+}$and supply $\mathrm{CO}_{2}$. Elimination of $\mathrm{H}^{+}$is done by the increased secretion of $\mathrm{H}^{+}$into the urine by the $\mathrm{Na}^{+}-\mathrm{H}^{+}$exchanger. As a result, reabsorption of $\mathrm{Na}^{+}$is boosted, and body fluid increases. The necessary $\mathrm{CO}_{2}$ in the RPT cytoplasm is obtained by the reaction (2) (oxaloacetate $\rightarrow$ phosphoenolpyruvate $+\mathrm{CO}_{2}$ ). This is a reaction in the cytoplasm. To move this reaction forward, it is necessary to expand the supply of oxaloacetate and reactivate phosphoenolpyruvate carboxykinase (PEPCK). Oxaloacetate is synthesized in the mitochondria through two reactions: a reaction that is caused by the catalysis of pyruvate carboxylase from pyruvate [reaction (1)], and a reaction that is caused by way of the tricarboxylic acid (TCA) cycle [reaction (4)]. The oxaloacetate synthesized in mitochondria is moved to the cytoplasm through the malate shuttle. Under acidotic conditions, pyruvate carboxylase activity is increased, the reaction (1) (pyruvate $\rightarrow$ oxaloacetate) is enhanced. Under reduced glycolysis conditions, therefore, some GAAs that can be used for pyruvate synthesis are used, so the secretion of these GAAs into the urine may be decreased. To rotate the TCA cycle under these circumstances and increase the production of oxaloacetate, it is necessary to enhance the reaction (4) ( $\alpha$-ketoglutarate $\rightarrow$ succinyl-CoA $\rightarrow$ fumarate $\rightarrow$ oxaloacetate). Since some GAAs that can be used for synthesizing each of these substances are used, the urinary excretion of these GAAs is sure to decrease. An important reaction for this $\alpha$-ketoglutarate synthesis is "glutamine $\rightarrow$ glutamate + ammonia $\left(\mathrm{NH}_{3}\right)$, glutamate $\rightarrow \alpha$-ketoglutarate $+\mathrm{NH}_{3}$." [ reaction (5)] In acidosis, both the $\mathrm{H}^{+}$that is filtered, and the $\mathrm{H}^{+}$that is secreted, have increased. This increased $\mathrm{NH}_{3}$ production accelerates the urinary excretion of $\mathrm{H}^{+}$because of the " $\mathrm{H}^{+}+\mathrm{NH}_{3} \rightarrow \mathrm{NH}_{4}{ }^{+}$" reaction. Urinary $\mathrm{pH}$ is adjusted always appropriately by secreting ammonia into the urine. 
male students who had undergone freshman health checkups at Tohoku University $(\mathrm{n}=3,651)$, individuals whose BMI exceeded 30 $\mathrm{kg} / \mathrm{m}^{2}$ were used as the subjects for our study.

\section{Definition of PUS (+) in the subjects}

Of the subjects selected for this study, the ones in whom reddish-brown cloudy urine and pink sediments produced from centrifugation could be confirmed macroscopically and those whose UA crystals or urates could be confirmed microscopically were diagnosed as PUS $(+)$. Subjects who presented no urinary abnormalities either macroscopically or microscopically, and in whom no UA crystals or urates could be confirmed microscopically were diagnosed as PUS $(-)$. The precipitation of UA was confirmed microscopically in many obese subjects with low UpH values, although this could not be confirmed macroscopically. Such cases were excluded from the analysis.

\section{Power analysis}

An average difference in $\mathrm{UpH}$ of 0.2 is anticipated between uric acid-excreting [PUS (+)] and non-uric acid-excreting [PUS (-)] males (Ogawa et al. 2015b). We therefore conducted a power analysis using a standard deviation of 0.4 , a power of 0.8 , and a standardized effect volume of 0.5 , and obtained the same number of samples for the [PUS $(+)$ ] group and [PUS $(-)$ ] groups $(\mathrm{n}=64)$. Assuming a dropout ratio of $10 \%$, we found that 70 subjects in each group would be required. Of the targeted subjects, we used the body mass index (BMI) as the exclusion factor and obtained 72 subjects in the PUS (+) group and 78 subjects in the PUS (-) group (total number of subjects $=150$ ). We then additionally measured the urinary urea nitrogen (UUN), UUA, and urinary AA excretion (UAA) levels in all the subjects, and compared these values between the PUS (+) and the PUS (-) group.

This cross-sectional study targeted only healthy students who were not undergoing any kind of drug therapy. The evaluation criteria were as follows: age, gender, BMI, systolic blood pressure (SBP), diastolic blood pressure (DBP), UpH, heart rate (HR), UUN, UUA, and UAA. The AAs measured are presented in Table 1 .

\section{Measurements}

In this study, we conducted investigations after minimizing the influence of age, gender, and BMI. Since UpH is vulnerable to fluctuations through the day, we used the urine taken early in the morning on an empty stomach after fasting for 12 hours, instead of spot urine.

The UpH was measured using an AX-4030 (ARKRAY, Inc., Kyoto, Japan), a fully automated urine analyzer with a preset testpaper. BUN and UUN were measured using the urease-GLDH/ICDH UV method. UUN was measured after eliminating ammonia by the ammonia elimination method. UA and UUA were measured using the uricase peroxidase method (Hitachi LABOSPECT 008).

For measuring the UAA, after the protein had been removed from the urine samples using $6 \%$ sulfosalicylic acid, the sample was subjected to derivatization using a MassTrak AAA reagent kit. This kit contains 6-aminoquinolyl-N-hydroxysuccinimidyl carbamate and AQC dissolved in acetonitrile, which is then mixed with the sample with added borate buffer. We then measured the AA concentration using UltraPerformance Liquid Chromatography (MassTrak ${ }^{\mathrm{TM}}$ Amino Acid Analysis, column $2.1 \times 150 \mathrm{~mm}$, ACQUITY UPLC ${ }^{\circledR}$ system, and Empower ${ }^{\mathrm{TM}} 2$ software) (UV $260 \mathrm{~nm}$ ).

This study complied with the Helsinki Declaration and was conducted with the approval of the Medical Ethics Committee of Tohoku University. All the participants provided their full informed consent.

Statistical analysis

Since all the measurement values showed a normal distribution (Shapiro-Wilk test), they were recorded in the form of mean \pm standard deviation. Numerical figures between the PUS (+) and PUS (-) groups were compared by dispersion analysis (ANOVA on Ranks) using either the Student's t-test (for samples that showed equal variances) or the Kruskal-Wallis method (for samples that did not show equal variances). Single regression tests were conducted using Spearman's rank-order correlation. We also conducted multiple regression analysis using $\mathrm{UpH}$ as the dependent variable; factors that showed significant single correlation with $\mathrm{UpH}$ were used as the independent variables. Logistic regression analysis was also performed using the presence or absence of PUS as the dependent variable; $\mathrm{UpH}$, age, BMI, SBP, DBP, and HR were used as the independent variables. In all cases, $p<0.05$ was considered to indicate statistical significance.

\section{Results}

Compared with the PUS (-) group, the PUS (+) group showed lower values for UpH, UUN, UUA, and for the urinary excretion of aspartic acid, ornithine, proline, hydroxyproline, isoleucine, hydroxylysine, leucine, and glutamate (Table 1). In a logistic regression analysis conducted using the presence or absence of PUS as the dependent variable and $\mathrm{UpH}$, age, BMI, SBP, DBP, and HR as the independent variables, only $\mathrm{UpH}$ was identified as an independent risk factor $(\beta=-0.1060567, p=0.031)$. In the logistic regression analysis conducted using PUS as the dependent variable and UpH, UUN, UUA, and the urinary excretion of aspartic acid, ornithine, proline, hydroxyproline, isoleucine, leucine, and glutamate as the independent variables, UUA $(\beta=-0.01, p=0.032)$ and the urinary excretion of proline $(\beta=-0.161, p=0.035)$, glutamate $(\beta=-0.143, p=0.024)$, and ornithine $(\beta=-0.178, p=0.004)$ were identified as independent risk factors. However, a decrease in UUA is caused by the precipitation of UUA. Therefore, this decrease in UUA appears to be the result of PUS, rather than its cause. We therefore eliminated UUA and again conducted logistic regression analysis. The following were identified as independent risk factors: $\mathrm{UpH}(\beta=-1.428, p=$ $0.013)$, and urinary excretion of hydroxyproline $(\beta=$ $-0.145, p=0.043)$, proline $(\beta=-0.166, p=0.027)$, glutamate $(\beta=-0.0126, p=0.039)$, and ornithine $(\beta=-0.201$, $p=0.001)$.

Since it appears likely that PUS is clinically linked to $\mathrm{UpH}$, we examined the correlation between $\mathrm{UpH}$ and other factors (Table 2). The results showed that $\mathrm{UpH}$ correlated positively with UUA and the excretion of glutamate, threonine, serine, glutamine, isoleucine, glycine, alanine, ornithine, valine, $\beta$-alanine, arginine, taurine, carnosine, aspartic acid, leucine, lysine, alpha-aminoadipic acid, phenylalanine, citrulline, histidine, 1-methylhistidine, 3-methylhistidine, and alpha-aminobutyric acid, but correlated negatively with HR and SBP. Therefore, a multiple regression analysis using $\mathrm{UpH}$ as the dependent variable 
Table 1. Comparison between the values of each factor in PUS (+) and PUS (-).

\begin{tabular}{|c|c|c|c|c|c|c|c|c|c|c|c|}
\hline \multirow{3}{*}{$\frac{\mathrm{n}}{\mathrm{UpH}}$} & & & & & \multicolumn{3}{|c|}{ PUS (-) } & \multicolumn{3}{|c|}{ PUS (+) } & \multirow[b]{2}{*}{$\mathrm{p}$} \\
\hline & & & 150 & & & 78 & & & 72 & & \\
\hline & & 5.86 & \pm & 0.39 & 5.94 & \pm & 0.36 & 5.78 & \pm & 0.40 & 0.012413 \\
\hline age & years & 18.52 & \pm & 1.40 & 18.55 & \pm & 1.65 & 18.48 & \pm & 1.07 & 0.776618 \\
\hline BMI & $\mathrm{kg} / \mathrm{m}^{2}$ & 24.13 & \pm & 4.10 & 24.07 & \pm & 4.18 & 24.20 & \pm & 4.04 & 0.843431 \\
\hline SBP & $\mathrm{mmHg}$ & 137.98 & \pm & 17.31 & 136.05 & \pm & 16.44 & 139.55 & \pm & 18.09 & 0.156266 \\
\hline DBP & $\mathrm{mmHg}$ & 76.68 & \pm & 12.59 & 75.15 & \pm & 13.38 & 78.13 & \pm & 11.54 & 0.122595 \\
\hline HR & bpm & 90.35 & \pm & 16.13 & 88.26 & \pm & 16.17 & 92.17 & \pm & 15.89 & 0.098713 \\
\hline UUN & $\mathrm{mmol} / \mathrm{g} \mathrm{Cr}$ & 170.57 & \pm & 49.68 & 180.10 & \pm & 52.01 & 160.24 & \pm & 45.13 & 0.013939 \\
\hline UUA & $\mu \mathrm{mol} / \mathrm{g} \mathrm{Cr}$ & 601.75 & \pm & 428.82 & 747.55 & \pm & 451.79 & 443.80 & \pm & 340.43 & $<0.00001$ \\
\hline taurine & $\mu \mathrm{mol} / \mathrm{g} \mathrm{Cr}$ & 587.66 & \pm & 398.91 & 610.78 & \pm & 372.25 & 568.56 & \pm & 427.14 & 0.461882 \\
\hline phosphatidylethanolamines & $\mu \mathrm{mol} / \mathrm{g} \mathrm{Cr}$ & 32.40 & \pm & 12.28 & 33.20 & \pm & 11.51 & 31.57 & \pm & 13.08 & 0.408799 \\
\hline aspartic acid & $\mu \mathrm{mol} / \mathrm{g} \mathrm{Cr}$ & 11.01 & \pm & 3.90 & 12.23 & \pm & 4.19 & 9.72 & \pm & 3.07 & 0.000041 \\
\hline hydroxy proline & $\mu \mathrm{mol} / \mathrm{g} \mathrm{Cr}$ & 7.98 & \pm & 3.27 & 8.79 & \pm & 3.33 & 7.12 & \pm & 2.98 & 0.001258 \\
\hline threonine & $\mu \mathrm{mol} / \mathrm{g} \mathrm{Cr}$ & 92.69 & \pm & 56.81 & 97.90 & \pm & 58.18 & 87.62 & \pm & 55.14 & 0.243621 \\
\hline serine & $\mu \mathrm{mol} / \mathrm{g} \mathrm{Cr}$ & 252.72 & \pm & 98.00 & 264.82 & \pm & 96.10 & 240.46 & \pm & 99.01 & 0.115823 \\
\hline asparagine & $\mu \mathrm{mol} / \mathrm{g} \mathrm{Cr}$ & 76.15 & \pm & 37.24 & 79.25 & \pm & 33.65 & 73.25 & \pm & 40.75 & 0.290431 \\
\hline glutamate & $\mu \mathrm{mol} / \mathrm{g} \mathrm{Cr}$ & 16.21 & \pm & 4.43 & 16.90 & \pm & 4.61 & 15.50 & \pm & 4.14 & 0.047222 \\
\hline glutamine & $\mu \mathrm{mol} / \mathrm{g} \mathrm{Cr}$ & 286.97 & \pm & 115.01 & 292.61 & \pm & 113.63 & 282.01 & \pm & 116.98 & 0.533569 \\
\hline sarcosine & $\mu \mathrm{mol} / \mathrm{g} \mathrm{Cr}$ & ND & & & ND & & & ND & & & - \\
\hline alpha-amino adipic acid & $\mu \mathrm{mol} / \mathrm{g} \mathrm{Cr}$ & 24.52 & \pm & 13.79 & 25.25 & \pm & 12.97 & 23.92 & \pm & 14.68 & 0.502364 \\
\hline proline & $\mu \mathrm{mol} / \mathrm{g} \mathrm{Cr}$ & 14.35 & \pm & 3.83 & 15.34 & \pm & 4.37 & 13.31 & \pm & 2.79 & 0.000847 \\
\hline glycine & $\mu \mathrm{mol} / \mathrm{g} \mathrm{Cr}$ & 610.86 & \pm & 360.52 & 635.00 & \pm & 317.17 & 584.69 & \pm & 402.90 & 0.395139 \\
\hline alanine & $\mu \mathrm{mol} / \mathrm{g} \mathrm{Cr}$ & 160.80 & \pm & 82.22 & 163.67 & \pm & 79.80 & 159.09 & \pm & 85.22 & 0.657018 \\
\hline citrulline & $\mu \mathrm{mol} / \mathrm{g} \mathrm{Cr}$ & 3.13 & \pm & 2.27 & 3.35 & \pm & 2.24 & 2.94 & \pm & 2.30 & 0.265382 \\
\hline alpha-amino butyric acid & $\mu \mathrm{mol} / \mathrm{g} \mathrm{Cr}$ & 8.21 & \pm & 3.59 & 7.75 & \pm & 3.51 & 8.72 & \pm & 3.64 & 0.107120 \\
\hline valine & $\mu \mathrm{mol} / \mathrm{g} \mathrm{Cr}$ & 25.18 & \pm & 6.28 & 26.13 & \pm & 6.24 & 24.21 & \pm & 6.21 & 0.054684 \\
\hline cystine & $\mu \mathrm{mol} / \mathrm{g} \mathrm{Cr}$ & 41.03 & \pm & 10.89 & 41.14 & \pm & 9.54 & 41.01 & \pm & 12.26 & 0.893554 \\
\hline methionine & $\mu \mathrm{mol} / \mathrm{g} \mathrm{Cr}$ & 6.32 & \pm & 3.73 & 6.32 & \pm & 3.18 & 6.34 & \pm & 4.27 & 0.996254 \\
\hline cystathionine & $\mu \mathrm{mol} / \mathrm{g} \mathrm{Cr}$ & 12.27 & \pm & 8.05 & 13.24 & \pm & 8.19 & 11.23 & \pm & 7.82 & 0.124624 \\
\hline isoleucine & $\mu \mathrm{mol} / \mathrm{g} \mathrm{Cr}$ & 9.54 & \pm & 2.56 & 10.18 & \pm & 2.64 & 8.88 & \pm & 2.30 & 0.001337 \\
\hline leucine & $\mu \mathrm{mol} / \mathrm{g} \mathrm{Cr}$ & 23.75 & \pm & 6.98 & 24.81 & \pm & 7.32 & 22.67 & \pm & 6.44 & 0.042363 \\
\hline tyrosine & $\mu \mathrm{mol} / \mathrm{g} \mathrm{Cr}$ & 56.52 & \pm & 24.60 & 57.02 & \pm & 25.36 & 56.21 & \pm & 23.90 & 0.798594 \\
\hline beta alanine & $\mu \mathrm{mol} / \mathrm{g} \mathrm{Cr}$ & 25.11 & \pm & 23.75 & 24.29 & \pm & 12.56 & 26.24 & \pm & 31.80 & 0.658776 \\
\hline phenylalanine & $\mu \mathrm{mol} / \mathrm{g} \mathrm{Cr}$ & 35.24 & \pm & 10.51 & 36.55 & \pm & 11.32 & 33.96 & \pm & 9.43 & 0.112312 \\
\hline beta-amino iso butyric acid & $\mu \mathrm{mol} / \mathrm{g} \mathrm{Cr}$ & 346.53 & \pm & 396.03 & 324.69 & \pm & 392.83 & 373.49 & \pm & 400.85 & 0.483926 \\
\hline homocysteine & $\mu \mathrm{mol} / \mathrm{g} \mathrm{Cr}$ & ND & & & ND & & & ND & & & - \\
\hline gamma amino butyric acid & $\mu \mathrm{mol} / \mathrm{g} \mathrm{Cr}$ & ND & & & $\mathrm{ND}$ & & & ND & & & - \\
\hline m-ethanol amine & $\mu \mathrm{mol} / \mathrm{g} \mathrm{Cr}$ & 276.04 & \pm & 63.41 & 278.47 & \pm & 67.70 & 272.86 & \pm & 58.76 & 0.626787 \\
\hline hydroxylysine & $\mu \mathrm{mol} / \mathrm{g} \mathrm{Cr}$ & 10.93 & \pm & 6.39 & 12.15 & \pm & 6.61 & 9.69 & \pm & 5.91 & 0.015117 \\
\hline ornithine & $\mu \mathrm{mol} / \mathrm{g} \mathrm{Cr}$ & 15.01 & \pm & 6.91 & 17.65 & \pm & 6.78 & 12.19 & \pm & 5.87 & $<0.00001$ \\
\hline 1 methyl histidine & $\mu \mathrm{mol} / \mathrm{g} \mathrm{Cr}$ & 461.39 & \pm & 503.56 & 464.82 & \pm & 514.95 & 462.83 & \pm & 494.51 & 0.931188 \\
\hline histidine & $\mu \mathrm{mol} / \mathrm{g} \mathrm{Cr}$ & 648.85 & \pm & 269.71 & 671.81 & \pm & 286.51 & 626.11 & \pm & 249.85 & 0.279325 \\
\hline lysine & $\mu \mathrm{mol} / \mathrm{g} \mathrm{Cr}$ & 127.09 & \pm & 122.30 & 131.84 & \pm & 103.71 & 122.14 & \pm & 140.26 & 0.622278 \\
\hline 3-methyl histidine & $\mu \mathrm{mol} / \mathrm{g} \mathrm{Cr}$ & 254.96 & \pm & 53.31 & 260.75 & \pm & 50.74 & 249.61 & \pm & 55.65 & 0.167670 \\
\hline tryptophan & $\mu \mathrm{mol} / \mathrm{g} \mathrm{Cr}$ & 44.87 & \pm & 16.68 & 45.06 & \pm & 16.90 & 44.90 & \pm & 16.56 & 0.888109 \\
\hline anserine & $\mu \mathrm{mol} / \mathrm{g} \mathrm{Cr}$ & 77.62 & \pm & 49.55 & 77.15 & \pm & 42.06 & 78.05 & \pm & 56.16 & 0.945190 \\
\hline carnosine & $\mu \mathrm{mol} / \mathrm{g} \mathrm{Cr}$ & 22.17 & \pm & 22.65 & 21.28 & \pm & 17.80 & 23.31 & \pm & 27.03 & 0.616475 \\
\hline arginine & $\mu \mathrm{mol} / \mathrm{g} \mathrm{Cr}$ & 18.73 & \pm & 5.48 & 19.30 & \pm & 4.38 & 18.20 & \pm & 6.43 & 0.192728 \\
\hline alloisoleucine & $\mu \mathrm{mol} / \mathrm{g} \mathrm{Cr}$ & ND & & & ND & & & ND & & & . \\
\hline
\end{tabular}

PUS, pink urine syndrome (precipitation of uric acid in the urine.); $\mathrm{UpH}$, urinary $\mathrm{pH}$; BMI, body mass index; SBP, systolic blood pressure; DBP, diastolic blood pressure; HR, heart rate; UUN, urinary urea nitrogen; UUA, urinary uric acid; $\mathrm{Cr}$, urinary creatinine excretion.

and ten factors (excluding UUA) starting from those with the smallest $p$-values as the dependent variables (from the above-mentioned glutamate to $\beta$-alanine; Table 3 ) was conducted, and only urinary glutamate excretion $(\beta=0.0387, p$
$<0.0001)$ was identified as an independent risk factor.

\section{Discussion}

PUS appears to be a phenomenon in which the synthe- 
Table.2. Correlations between $\mathrm{UpH}$ and various factors.

\begin{tabular}{|c|c|c|c|c|c|c|c|c|}
\hline & $\mathrm{UpH}$ & $\mathrm{p}$ & & $\mathrm{UpH}$ & $\mathrm{p}$ & & $\mathrm{UpH}$ & $\mathrm{p}$ \\
\hline age & -0.0242 & 0.7690 & glycine & 0.2813 & 0.0005 & 1 methyl histidine & 0.1794 & 0.0281 \\
\hline BMI & -0.1574 & 0.0544 & alanine & 0.2793 & 0.0005 & histidine & 0.1804 & 0.0272 \\
\hline SBP & -0.1908 & 0.0194 & citrulline & 0.1928 & 0.0240 & lysine & 0.2058 & 0.0115 \\
\hline DBP & -0.0992 & 0.2273 & alpha-amino butyric acid & 0.1627 & 0.0466 & 3-methyl histidine & 0.1714 & 0.0360 \\
\hline HR & -0.1950 & 0.0160 & valine & 0.2679 & 0.0009 & tryptophan & 0.0650 & 0.4291 \\
\hline UUN & 0.1290 & 0.1158 & cystine & 0.1281 & 0.1182 & anserine & 0.1633 & 0.2166 \\
\hline UUA & 0.3547 & $<0.0001$ & methionine & 0.1379 & 0.0924 & carnosine & 0.2370 & 0.0035 \\
\hline taurine & 0.2455 & 0.0026 & cystathionine & 0.1092 & 0.1849 & arginine & 0.2607 & 0.0013 \\
\hline phosphatidylethanolamines & -0.1555 & 0.0574 & isoleucine & 0.2895 & 0.0003 & alloisoleucine & - & - \\
\hline aspartic acid & 0.2344 & 0.0039 & leucine & 0.2059 & 0.0115 & & & \\
\hline hydroxy proline & 0.1029 & 0.2102 & tyrosine & 0.0956 & 0.2447 & & & \\
\hline threonine & 0.3084 & 0.0001 & $\mathrm{~b}$ alanine & 0.2651 & 0.0010 & & & \\
\hline serine & 0.3226 & 0.0001 & phenylalanine & 0.1982 & 0.0150 & & & \\
\hline asparagine & 0.4064 & $<0.0001$ & beta-amino iso butyric acid & 0.0073 & 0.9292 & & & \\
\hline glutamate & 0.3884 & $<0.0001$ & homocysteine & - & - & & & \\
\hline glutamine & 0.3028 & 0.0002 & gamma amino butyric acid & - & - & & & \\
\hline sarcosine & - & - & m-ethanol amine & 0.0389 & 0.6363 & & & \\
\hline alpha-amino adipic acid & 0.2003 & 0.0140 & hydroxylysine & 0.1275 & 0.1199 & & & \\
\hline proline & 0.1194 & 0.1457 & ornithine & 0.2811 & 0.0005 & & & \\
\hline
\end{tabular}

$\mathrm{UpH}$, urinary pH; BMI, body mass index; SBP, systolic blood pressure; DBP, diastolic blood pressure; HR, heart rate; UUN, urinary urea nitrogen; UUA, urinary uric acid.

Table 3. Correlation coefficient between $\mathrm{UpH}$ and the factors which are related with $\mathrm{UpH}$, starting from those with the smallest $p$-values.

\begin{tabular}{|c|c|c|c|c|c|}
\hline & $\mathrm{UpH}$ & $p$ & & $\mathrm{UpH}$ & $p$ \\
\hline UUA & 0.3547 & $<0.0001$ & taurine & 0.2455 & 0.0026 \\
\hline asparagine & 0.4064 & $<0.0001$ & carnosine & 0.2370 & 0.0035 \\
\hline glutamic acid & 0.3884 & $<0.0001$ & aspartic acid & 0.2344 & 0.0039 \\
\hline threonine & 0.3084 & 0.0001 & lysine & 0.2058 & 0.0115 \\
\hline serine & 0.3226 & 0.0001 & leucine & 0.2059 & 0.0115 \\
\hline glutamine & 0.3028 & 0.0002 & alpha-amino adipic acid & 0.2003 & 0.0140 \\
\hline isoleucine & 0.2895 & 0.0003 & phenylalanine & 0.1982 & 0.0150 \\
\hline glycine & 0.2813 & 0.0005 & SBP & -0.1908 & 0.0194 \\
\hline alanine & 0.2793 & 0.0005 & citrulline & 0.1928 & 0.0240 \\
\hline ornithine & 0.2811 & 0.0005 & histidine & 0.1804 & 0.0272 \\
\hline valine & 0.2679 & 0.0009 & 1 methyl histidine & 0.1794 & 0.0281 \\
\hline beta alanine & 0.2651 & 0.0010 & 3-methyl histidine & 0.1714 & 0.0360 \\
\hline arginine & 0.2607 & 0.0013 & alpha-amino butyric acid & 0.1627 & 0.0466 \\
\hline
\end{tabular}

$\mathrm{UpH}$, urinary $\mathrm{pH}$; UUA, urinary uric acid; $\mathrm{SBP}$, systolic blood pressure.

sis of $\mathrm{NH}_{3}$ from glutamate decreases, $\mathrm{UpH}$ reduces, and UA precipitates into the urine. We therefore predicted that, in PUS, together with a drop in $\mathrm{UpH}$, the amount of urinary glutamate excreted would increase. We indeed found that the amount of urinary glutamate excreted was decreased to such a level that it reduced UpH. In other words, although glutamate was being actively reabsorbed and used by the RPTs for decreasing the amount excreted into the urine, it was not being used for $\mathrm{NH}_{3}$ synthesis. What, then, is this reabsorbed glutamate used for?
We observed that a reduction in $\mathrm{UpH}$ was strongly related to the increased OS (Ogawa et al. 2015a, b). When OS increases, the body invariably reacts in an attempt to counteract it. One such reaction is the nuclear factor-E2 p45-related factor (Nrf) 2-mediated OS response (Kwak et al. 2004; Sun et al. 2007). When OS increases, intracellular Nrf2 is activated and moves to within the nucleus to exert transcriptional activity. Nrf2 increases the synthesis of UA, lactic acid, and glutathione in an effort to eliminate OS (Mitsuishi et al. 2012). It is believed that, since glutamate 
is used for this glutathione synthesis, the supply of glutamate for the $\mathrm{NH}_{3}$ synthesis reaction is reduced, thereby slowing $\mathrm{NH}_{3}$ synthesis (Fig. 2).

The next question that we aimed to answer was that if the glutamate in the RPTs is depleted, could the RPTs cope by increasing glutamate reabsorption? The rate of glutamate reabsorption in the RPTs is close to $100 \%$ to begin with; therefore, there is very little scope for further increase (around $1 \%$ at the most). Thus, there is a limit to the increased reabsorption that results from the increased demand for glutamate. Consequently, if the major share of the reabsorbed glutamate is consumed for glutathione synthesis, the amount of glutamate available for $\mathrm{NH}_{3}$ synthesis is likely to decrease. Unfortunately, since we did not measure glutathione or urinary $\mathrm{NH}_{4}^{+}$in this study, we were unable to confirm any such changes in glutathione or reductions in $\mathrm{NH}_{3}$ levels; these topics should be explored in future studies. Urinary $\mathrm{NH}_{4}^{+}$is reported to decrease in association with diabetes, in which increased OS is observed (Maalouf et al. 2010). In our report, we also noted that $\mathrm{UpH}$ correlated negatively with urinary OS markers (Ogawa et al. 2015b).

It also appears unlikely that UA precipitation occurs due to decreased $\mathrm{UpH}$ levels alone, leading us to believe that an increase in UA synthesis and UUA excretion are involved in UA precipitation. In PUS, however, since UA is already precipitated into the urine, its UUA concentration is low, making it impossible to evaluate the level of UUA excretion. This, too, appears to be an obstacle that needs to be overcome in further studies. In our previous study targeting diabetes patients, we found that the serum UA concentration correlates negatively with $\mathrm{UpH}$ (Ogawa et al. 2015a).

Moreover, although lactic acid synthesis increases with

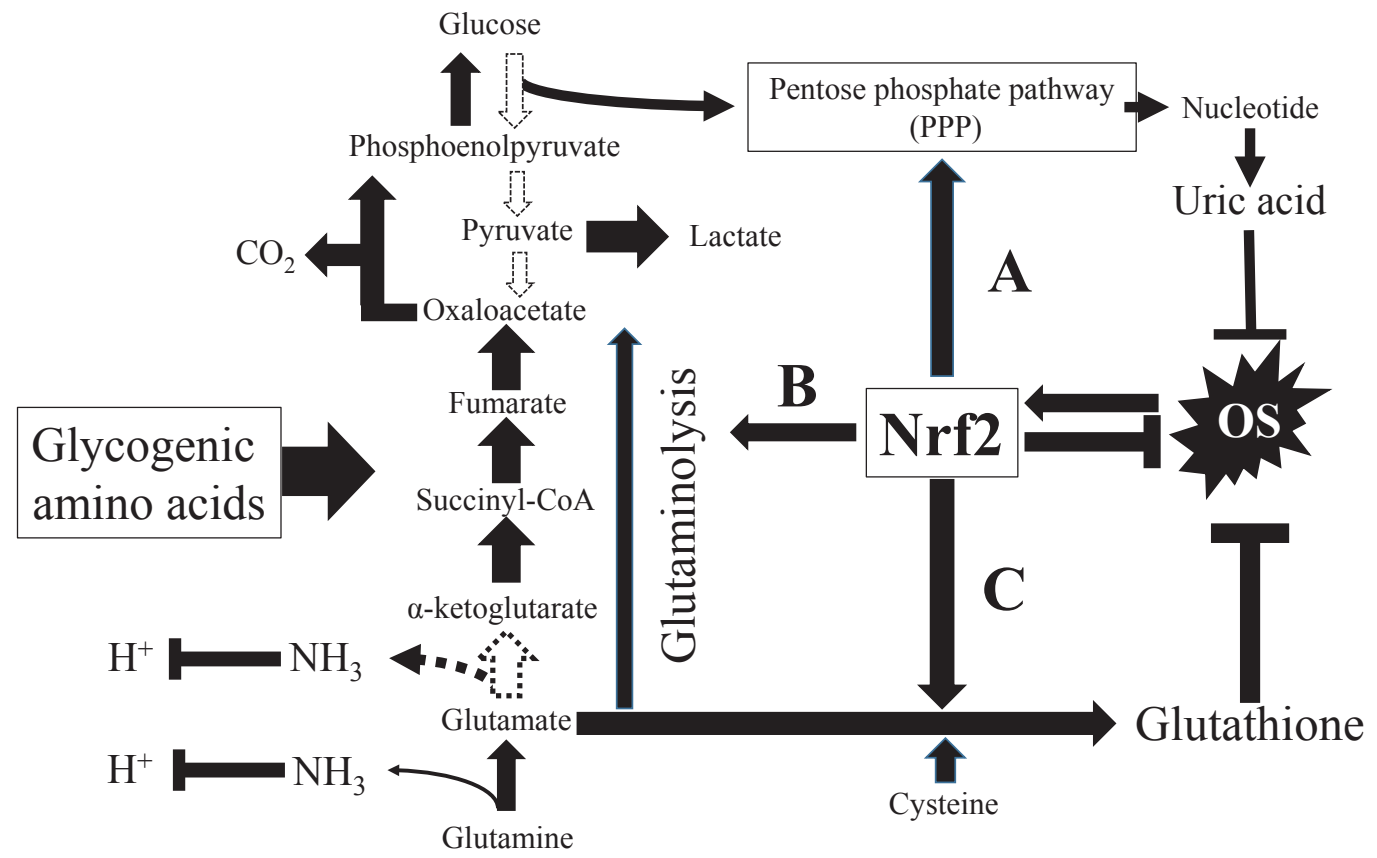

Fig. 2. Relations between synthesis of ammonia and $\mathrm{Nrf} 2$ and glucogenic amino acids.

If oxidative stress (OS) increases in the renal proximal tubules (RPT), nuclear factor-E2 p45-related factor (Nrf) 2 becomes reactivated to counteract such OS increases.

Nrf2 increases nucleic acid synthesis via the pentose phosphate pathway, increases the production of UA (A), promotes the synthesis of oxaloacetate from $\alpha$-ketoglutarate (B), and increases the conversion of glutamate to glutathione (C).

If these actions of Nrf2 are taken into consideration, it becomes easier to explain the following:

A: increased uric acid (UA) production; Nrf2 also activates the pentose phosphate pathway, and increases the production of UA.

B: increased reabsorption of glucogenic amino acids (GAAs); Pink urine syndrome (PUS) $(+)$ subjects showed a greater reduction in the amount of urinary excretion of some GAAs than PUS (-) subjects. This shows that many GAAs are reabsorbed in the RPT and are used actively. The GAAs are used as a substrate of oxaloacetate, fumarate and succinyl CoA syntheses. This reaction is activated by Nrf2 and the lactic acid is synthesized finally.

Nrf2 promotes the glutaminolysis "glutamate $\rightarrow \alpha$-ketoglutarate $\rightarrow$ succinyl-CoA $\rightarrow$ fumarate $\rightarrow$ oxaloacetate $\rightarrow$ phosphoenolpyruvate $\rightarrow$ pyruvate $\rightarrow$ lactate" reaction, so large amounts of amino acids such as GAAs are consumed. As a result, the amount of GAAs excreted from the kidneys decreases even further.

C: reduced production of ammonia $\left(\mathrm{NH}_{3}\right)$ from glutamate and decrease in $\mathrm{UpH}$. $\mathrm{NH}_{3}$ synthesis is comprised of two reactions: the synthesis of glutamate from glutamine, and the synthesis of $\alpha$-ketoglutarate from glutamate. As a result of this reactivation of $\mathrm{Nrf2}$, glutamate, not glutamine, is used for glutathione synthesis, so production of $\mathrm{NH}_{3}$ decreases, which in turns lowers the urinary $\mathrm{pH}$. Under these conditions, the production of $\mathrm{NH}_{3}$ is dependent on the synthesis of $\alpha$-ketoglutarate from glutamate, so the relationship between urinary $\mathrm{pH}$ and urinary glutamate excretion becomes extremely strong. 
the increased transcriptional activity of $\mathrm{Nrf} 2$, it is caused the increase in lactic acid synthesis caused by Nrf2. This lactic acid synthesis uses glutamine, glutamate, $\alpha$-ketoglutarate, succinyl CoA, fumarate, and oxaloacetate as the substrates (Mitsuishi et al. 2012) (Fig. 2). However, in situations where, because of Nrf2 activation, glutamate is consumed for fuel glutathione synthesis and glucose use has also decreased (insulin resistance), these substrates are replaced by glucogenic amino acids (GAAs) (Krebs et al. 1965; Stumpf and Kraus 1977; Jans and Willem 1991; Atlante et al. 1998; Mitsuishi et al. 2012; Kazubek-Zemke et al. 2014) (Fig. 1). Therefore, a reduction in $\mathrm{UpH}$ shows a positive correlation with a reduction in the amount of urinary excretion of various kinds of GAAs. Thus, reduced $\mathrm{NH}_{3}$ synthesis, which is attributable to a shortage of glutamate, is the direct cause of reduced $\mathrm{UpH}$; however, a reduction in the urinary excretion of other GAA types is the result of a different Nrf2 reaction. Thus, in multiple regression analysis, only the amount of glutamate excreted in the urine can be confidently identified as an independent risk factor for $\mathrm{UpH}$.

It is extremely easy to explain PUS as a group of phenomena that include (1) increased UA synthesis due to Nrf2 activation induced by increased OS in the RPTs, (2) increased use of GAAs associated with an increase in lactic acid synthesis, and (3) reduction in $\mathrm{NH}_{3}$ synthesis caused by the depletion of glutamate that results from increased glutathione synthesis (Mitsuishi et al. 2012) (Figs. 1 and 2). However, in this study, we identified that, in PUS, even a reduction in $\mathrm{UpH}$ is strongly related to a reduction in urinary glutamate excretion and that a reduction in $\mathrm{UpH}$ is correlated with a reduction in the urinary excretion of some GAAs. In future studies on this subject, Nrf2 activity, glutathione levels, reabsorption rate of various AAs, urinary $\mathrm{NH}_{4}{ }^{+}$, and blood lactic acid levels should also be measured.

In view of the above findings, it appears that PUS is a condition where OS increases in the RPTs due to obesity and other factors (Ogawa et al. 2015b), and where Nrf2 activity is increased to counteract such increased OS. A lower UpH itself increases OS in the RPTs (Souma et al. 2011), indicating a possibility that lowered UpH and increased OS in the RPTs may create a vicious cycle. In diabetes, moreover, a reduction in $\mathrm{UpH}$ is related to persistent renovascular disorders (Ogawa et al. 2015a). We believe that even the smallest reduction in $\mathrm{UpH}$ in obese and diabetic patients should be paid attention to.

Although PUS is observed in a large number of obese patients and cases with reduced UpH (Ogawa et al. 2015b), obesity and low $\mathrm{UpH}$ values are not essential for the development of PUS, neither do all obese cases necessarily show low UpH values or high UUA excretion (Strohmaier et al. 2012; Ogawa et al. 2015b). UA precipitation into the urine appears to occur as a result of a complex interaction between reduced $\mathrm{UpH}$ and increased urinary UA excretion. A reduction in $\mathrm{UpH}$ can occur either due to an increased supply of $\mathrm{H}^{+}$(acidosis) or a reduction in $\mathrm{H}^{+}$-eliminating capabilities (reduced $\mathrm{NH}_{3}$ synthesis); likewise, an increase in UUA excretion can occur either due to increased UA production or a reduction in UA reabsorption. In obese patients, several of these phenomena are likely to occur simultaneously (Strohmaier et al. 2012). Moreover, increased OS in the RPTs may occur not only in people with obesity but also in those with diabetes and renal diseases (Kazubek-Zemke et al. 2014; Ogawa et al. 2015b). PUS, therefore, is a phenomenon where the precipitation of UA into the urine is induced as a result of a complex combination of the effect of various factors in vivo.

In this study, we were unable to measure the urinary levels of $\mathrm{NH}_{3}$ and $\mathrm{NH}_{4}{ }^{+}$or the local production of $\mathrm{CO}_{2}$ in the kidneys. We also did not evaluate any changes in arterial plasma levels, AV-differences, or renal metabolism of the likely precursors for renal ammoniagenesis. Furthermore, we did not consider the diet of the subjects. No arterial blood gas analysis was conducted either. This was a study conducted in conjunction with health checkups of university students; therefore, it was not possible to carry out all these investigations. Moreover, since the expression of $\mathrm{Nrf}_{2}$ in the RPT has not been evaluated, our discussions have not been scientifically validated. Further validations with animal experiments are required to confirm our findings.

\section{Acknowledgments}

The authors wish to thank Miss Asako Chiba and Mrs. Yasuko Sato for their assistance with gathering the urine samples for the study. We also wish to thank the nurses, Eiko Mitsui, Yoko Hasegawa, Michiko Ota, Megumi Ito, and Hiromi Sato, for their efforts in gathering student data. Our deep appreciation also goes to all the members of the staff who helped us in this study.

This work was supported by Tohoku University's Center for the Advancement of Higher Education President's Research Fund.

\section{Author Contributions}

S.O. wrote the manuscript and analyzed the data, J.T., M.S., K.N., and M.O. contributed to collection of the samples, the discussion, and data analysis. S.I. reviewed and edited the manuscript.

\section{Conflict of Interest}

The authors declare no conflict of interest.

\section{References}

Atlante, A., Gagliardi, S. \& Passarella, S. (1998) Fumarate permeation in normal and acidotic rat kidney mitochondria: fumarate/malate and fumarate/aspartate translocators. Biochem. Biophys. Res. Commun., 243, 711-718.

Deitel, M., Thompson, D.A., Saldanha, C.F., Ramshaw, P.J., Patterson, M.C. \& Pritzker, K.P. (1984) "Pink urine" in morbidly obese patients following gastric partitioning. Can. Med. Assoc. J., 130, 1007-1011.

Hems, D.A. (1975) Biochemical aspects of renal ammonia formation in metabolic acidosis. Enzyme, 20, 359-380.

Jans, A.W. \& Willem, R. (1991) Metabolism of [2-13C] succinate in renal cells determined by 13C NMR. Eur. J. Biochem., 195, 
97-101.

Karim, Z., Szutkowska, M., Vernimmen, C. \& Bichara, M. (2005) Renal handling of NH3/NH4+: recent concepts. Nephron Physiol., 101, 77-81.

Kazubek-Zemke, M., Rybka, J., Marchewka, Z., Rybka, W., Pawlik, K. \& Długosz, A. (2014) Preliminary study on application of urine amino acids profiling for monitoring of renal tubular injury using GLC-MS. Postepy Hig. Med. Dosw. (Online), 68, 1299-1311.

Krebs, H.A., Speake, R.N. \& Hems. R. (1965) Acceleration of renal gluconeogenesis by ketone bodies and fatty acids. Biochem. J., 94, 712-720.

Kwak, M.K., Wakabayashi, N. \& Kensler, T.W. (2004) Chemoprevention through the Keap1-Nrf2 signaling pathway by phase 2 enzyme inducers. Mutat. Res., 555, 133-148.

Maalouf, N.M., Cameron, M.A., Moe, O.W. \& Sakhaee, K. (2010) Metabolic basis for low urine $\mathrm{pH}$ in type 2 diabetes. Clin. J. Am. Soc. Nephrol., 5, 1277-1281.

Mitsuishi, Y., Taguchi, K., Kawatani, Y., Shibata, T., Nukiwa, T., Aburatani, H., Yamamoto, M. \& Motohashi, H. (2012) Nrf2 redirects glucose and glutamine into anabolic pathways in metabolic reprogramming. Cancer Cell, 22, 66-79.

Moret, C., Dave, M.H., Schulz, N., Jiang, J.X., Verrey, F. \& Wagner, C.A. (2007) Regulation of renal amino acid transporters during metabolic acidosis. Am. J. Physiol. Renal. Physiol., 292, F555-566.

Nissim, I. (1999) Newer aspects of glutamine/glutamate metabolism: the role of acute $\mathrm{pH}$ changes. Am. J. Physiol., 277, F493-497.

Ogawa, S., Nako, K., Okamura, M. \& Ito, S. (2015a) Lower urinary $\mathrm{pH}$ is useful for predicting renovascular disorder onset in patients with diabetes. BMJ Open Diabetes Res. Care, 3, e000097.

Ogawa, S., Takiguchi, J., Nako, K., Okamura, M., Sakamoto, T., Kabayama, S., Mori, T., Kinouchi, Y. \& Ito, S. (2015b) Elucidation of the etiology and characteristics of pink urine in young healthy subjects. Clin. Exp. Nephrol., 19, 822-829.

Rodriguez-Nichols, F., Laughrey, E. \& Tannen, R.L. (1984) Response of renal NH3 production to chronic respiratory acidosis. Am. J. Physiol., 247, F896-903.

Souma, T., Abe, M., Moriguchi, T., Takai, J., YanagisawaMiyazawa, N., Shibata, E., Akiyama, Y., Toyohara, T., Suzuki, T., Tanemoto, M., Abe, T., Sato, H., Yamamoto, M. \& Ito, S. (2011) Luminal alkalinization attenuates proteinuria-induced oxidative damage in proximal tubular cells. J. Am. Soc. Nephrol., 22, 635-648.

Strohmaier, W.L., Wrobel, B.M. \& Schubert, G. (2012) Overweight, insulin resistance and blood pressure (parameters of the metabolic syndrome) in uric acid urolithiasis. Urol. Res., 40, 171-175.

Stumpf, B. \& Kraus, H. (1977) Gluconeogenesis in kidney cortex slices of the guinea pig. Its relation to acidosis and to calcium. Curr. Probl. Clin. Biochem., 8, 329-335.

Sun, Z., Zhang, S., Chan, J.Y. \& Zhang, D.D. (2007) Keap1 controls postinduction repression of the Nrf2-mediated antioxidant response by escorting nuclear export of Nrf2. Mol. Cell. Biol., 27, 6334-6349.

Tapiero, H., Mathé, G., Couvreur, P. \& Tew, K.D. (2002) II. Glutamine and glutamate. Biomed. Pharmacother., 56, 446-457.

Zipp, T. \& Tannen, R.L. (1983) Influence of phosphoenolpyruvate carboxykinase inhibition on the response of $\mathrm{NH} 3$ production to acute acidosis. J. Lab. Clin. Med., 102, 198-204. 